
allemande

51-1 | 2019

La République démocratique allemande dans l'espace public européen (1949-2018)

\title{
Grenzüberschreitungen. Das DDR-Fernsehen im europäischen Kontext - das Beispiel Frankreich
}

Thomas Beutelschmidt

\author{
(2) OpenEdition \\ Journals \\ Édition électronique \\ URL : https://journals.openedition.org/allemagne/1377 \\ DOI : $10.4000 / a l l e m a g n e .1377$ \\ ISSN : 2605-7913 \\ Éditeur \\ Société d'études allemandes \\ Édition imprimée \\ Date de publication : 2 juillet 2019 \\ Pagination : 67-81 \\ ISSN : 0035-0974 \\ Référence électronique \\ Thomas Beutelschmidt, "Grenzüberschreitungen. Das DDR-Fernsehen im europäischen Kontext - das \\ Beispiel Frankreich", Revue d'Allemagne et des pays de langue allemande [Online], 51-1 | 2019, Online \\ erschienen am: 02 Juli 2020, abgerufen am 02 Juni 2022. URL: http://journals.openedition.org/ \\ allemagne/1377 ; DOI: https://doi.org/10.4000/allemagne.1377
}




\section{Grenzüberschreitungen. Das DDR-Fernsehen im europäischen Kontext - das Beispiel Frankreich}

\section{- Thomas Beutelschmidt*}

Der Beitrag möchte einen weiteren Beleg dafür liefern, dass die DDR selbst in Zeiten des Kalten Kriegs keineswegs hermetisch von Westeuropa abgeschnitten war - auch wenn sie stets darauf bedacht war, sich mit hoher Anpassungsbereitschaft als, Musterland' in die sozialistische Staatengemeinschaft zu integrieren und weitgehend von der westlichen Wertegemeinschaft sowie insbesondere der konkurrierenden Referenzgesellschaft in der Bundesrepublik abzuschotten.

Vor allem im Äther und damit im Bereich der Funkmedien gelang die Abgrenzung weniger lückenlos. Zwar unterlagen Radio und Fernsehen weitreichenden Kontrollmechanismen und galten als eine wichtige „Waffe“ in der ideologischen Systemauseinandersetzung. Aber trotz der Trennungsgeschichte sind grenz- und systemübergreifende Interaktionen und Verflechtungen früh erkennbar. Bereits in den 1960er Jahren konstatierten Kommunikationswissenschaftler wie Ithiel de Sola Pool: „The iron curtain is a porous one “(1). Und Jean d'Arcy prophezeite etwa zeitgleich als Programmdirektor des französischen Fernsehens: „The ,television without frontiers' may not be far off“(2), was sich auf ganz Europa bezog. Man kann vielleicht von „connected enemies“ ${ }^{\text {(3) }}$ sprechen, deren Beziehungen sowohl von makropolitischer Konfliktlogik als auch von medienimmanenter Dynamik geprägt waren.

* Dr. Thomas Beutelschmidt, Medienhistoriker und Publizist, ist assoziierter Mitarbeiter am Zentrum für Zeitgenössische Forschung Potsdam (ZZF).

1 Zitiert in James Sсншосн, Global TV. New Media and the Cold War. 1946-69, Champaign, University of Illinois, 2009, S. 117.

2 Jean d'Arcy, Working Paper UNESCO: Television in the Service of international Understanding, Paris, 16.06.1960, S. 1 .

3 Thomas Beutelschmidt, Richard Oenmig, „Connected Enemies? Programming Transfer between East and West during the Cold War and the Example of East German Television“, View, n5 (2014), S. 60-67. 
Im Kontext des Cold War Culture-Diskurses erscheint die ostdeutsche Fernsehlandschaft besonders aussagekräftig. Zum einen kam der DDR im kontinentalen Medienverkehr als Transitland an der Nahtstelle und Bruchzone der Machtblöcke eine wichtige Rolle zu. Zum anderen wurde der inter- und transnationale Programmtransfer nach Ost wie nach West rege genutzt. Das DDR-Fernsehen wurde also von unterschiedlichen TV-Kulturen aus beiden Lagern gespeist und musste sich in einem Spannungsfeld zwischen sozialistischer Nationalkultur und Globalisierung behaupten. So konstatierte der DDR-Publizist Robert Michel schon 1969:

„Im Grunde genommen arbeiten die Fernsehschaffenden der DDR von Beginn an unter modellhaften Bedingungen des Kontinental- oder Globalfernsehens. Sie mussten, ob sie wollten oder nicht, ihre Erfahrungen machen mit einem Empfang über staatliche Grenzen hinweg“(4).

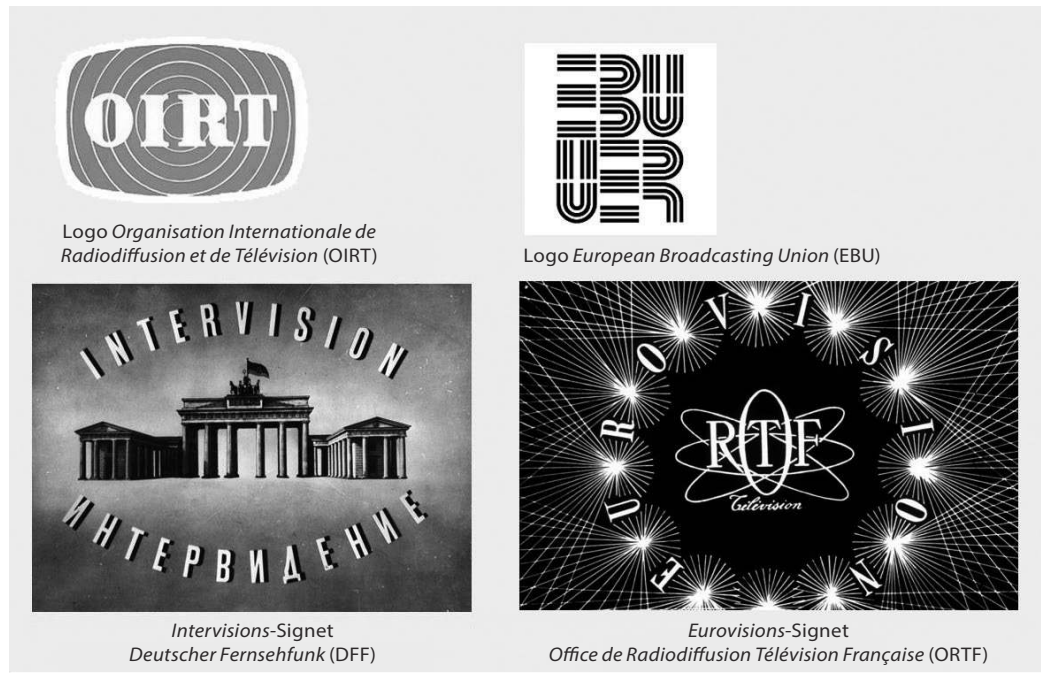

Abb. 1: Erkennungszeichen in Ost und West (Quelle: OIRT/EBU)

Der multilaterale Austausch erfolgte über den Dachverband der Organisation Internationale de Radiodiffusion et de Télévision (OIRT) ${ }^{(5)}$ in Prag. Er war ursprünglich als Organisation Internationale de Radiodiffusion (OIR) am 28. Juni 1946 in Brüssel als gesamteuropäische Initiative gegründet worden. Wegen politischer Unvereinbarkeit verließen jedoch die westlichen Rundfunkeinrichtungen die OIR und gründeten am 12. Februar 1950 als Alternative den Europäischen Rundfunkverein (EBU/UER) mit Verwaltungssitz in Genf und dem technischen Zentrum in Brüssel. Das Scheitern und eine separate Einrichtung auf Initiative der British Broadcasting Corporation (BBC) resultierten nicht nur aus den unterschiedlichen Positionen in Bezug auf die Neuverteilung

4 Robert Michel, Die Makrostruktur der Rezeptionsbedingungen des DDR-Fernsehens und einige programmpolitische Folgerungen, insbesondere für den journalistischen Bereich, Diss. phil., Sektion Journalistik, Karl-Marx-Universität Leipzig, 1969, S. 54.

5 Zum Selbstverständnis, zur Struktur und zu den Aufgaben, siehe Thomas Beutelschmidt, OSTWEST-GLOBAL. Das sozialistische Fernsehen im Kalten Krieg, Leipzig, Vistas, 2017. 
der verfügbaren Frequenzen. Sie sind ebenso auf die unüberbrückbaren Gegensätze zwischen den Mitgliedern unter sowjetischem Einfluss (zu diesem Zeitpunkt nominell 16) und den demokratischen Staaten (nur 12 Vertreter) zurückzuführen. Die erfolgte Spaltung in zwei Organisationen kann als eine Aufteilung und Fraktionierung der Rundfunklandschaft gelten, die als „halbierte Globalisierung “(6) dem Antagonismus des Kalten Krieges entsprach. Damit kennzeichnet diese Entwicklung im Medienbereich das, was sich parallel mit der Warschauer Vertragsorganisation (WVO/,Warschauer Pakt") und der North Atlantic Treaty Organization (NATO) auf militärischem bzw. mit dem Rat für gegenseitige Wirtschaftshilfe (RGW) und der Organisation für europäische wirtschaftliche Zusammenarbeit (OEEC) auf wirtschaftlichem Gebiet vollzogen hat.

In der Folge koordinierte die OIR(T) einerseits die Zusammenarbeit der Radio- und Fernseheinrichtungen in der sozialistischen Staatengemeinschaft. Andererseits vertrat sie deren Interessen gegenüber den anderen Rundfunkorganisationen wie der EBU/UER und ihrem Eurovision-System als Zusammenschluss der westlichen TV-Nationen.

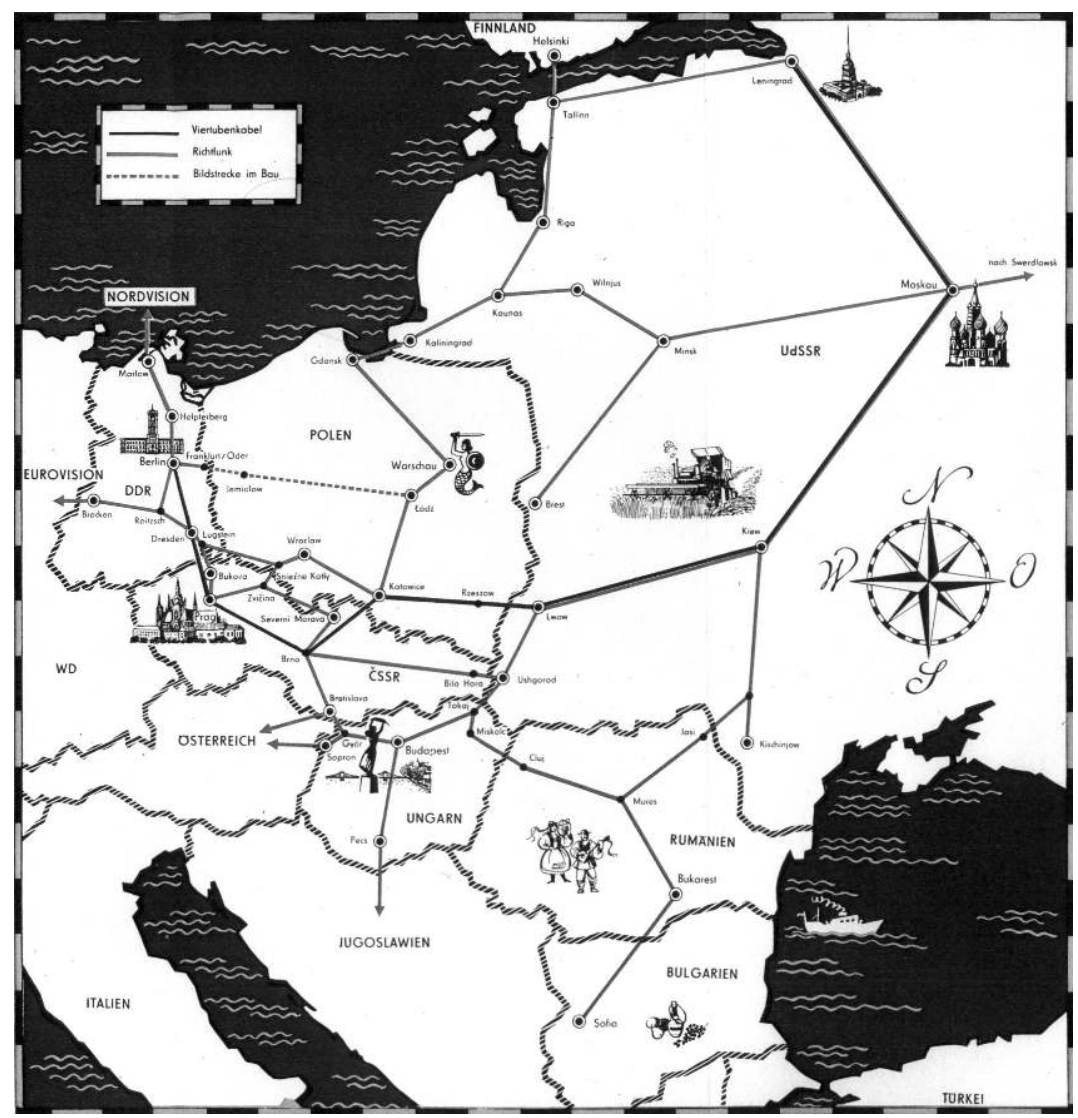

Abb. 2: TV-Strecken in Osteuropa für den bi- und multilateralen Programmaustausch (Quelle: OIRT/Grafik DFF, Presseabteilung 1964)

6 Jürgen Osterhammel, Niels P. Petersson, Geschichte der Globalisierung. Dimensionen. Prozesse. Epochen, München, C.H. Beck, 2003. 
Die ostdeutschen Funkmedien hatte die OIR 1951 in ihre Reihen aufgenommen. Die DDR war zudem 1960 Gründungsmitglied des Distributionsnetzes Intervision und seit 1972 der Intersputnik-Organisation für die Satellitenkommunikation angeschlossen. Damit wurde sie auch auf dem Gebiet der Medien fest in das „sozialistische Weltsystem“ integriert.

Dank des weitgespannten Kommunikationsnetzes konnte das Telemedium auch im Osten seinen Anspruch als ,Fenster zur Welt ${ }^{\star}$ einlösen und zumindest eine kontrollierte Teilhabe am aktuellen Geschehen versprechen. Mit ihrem Verband hatten die Ostblock-Länder zudem ein funktionierendes Steuerungs- und Propagandainstrument zur Verfügung, mit dem sie über ausgewählte Bilder auch im Westen ihre Botschaften kontrolliert verbreiten konnten.

Selbst wenn hinter den Aktivitäten der OIRT vor allem der sowjetische Hegemonialanspruch stand, so wurden von den osteuropäischen Partnern bei aller Abhängigkeit und Instrumentalisierung auch Eigeninteressen formuliert und durchgesetzt. Denn die Television ist grundsätzlich als ausdifferenziertes Mediensystem mit immanenter Eigenrationalität zu betrachten, das einen relativen Anspruch auf mediale Selbstbestimmung in den Koordinaten der Parteidoktrin erkennen lässt.

Am Beispiel des DDR-Fernsehens möchte ich im Folgenden die westlichen Außenbeziehungen und ihre Auswirkungen auf das eigene Medienverständnis betrachten. Die externen Verbindungen und die sichtbare Medienpräsenz erwiesen sich als eine „vierte Dimension der Außenpolitik“(7) im Kampf um die völkerrechtliche Anerkennung als durchaus produktiv. Ihre außerhalb des Sowjetblocks lange ignorierte

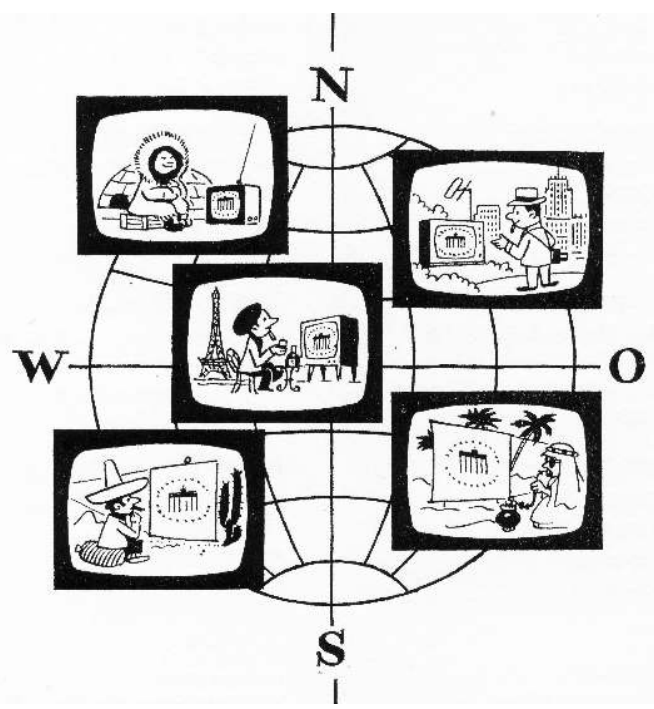

Abb. 3: DDR-Fernsehen global seit Mitte der 1960er Jahre (Quelle: Grafik DFF/DDR TV international 2/1966/DRA SGFS)
Souveränität hielt die DDR in einem permanenten Aktionsmodus: Zum einen durch forcierten Programmexport, zum anderen über die Mitarbeit in den internationalen Organisationen wie u.a. in der Fernmeldunion (UIT/ ITU) als Sonderorganisation der Vereinten Nationen für die weltweite Regulierung der technischen Infrastruktur im Rundfunkwesen.

Mit Nachdruck signalisierte der ewige Paria seine angebliche Modernität und Weltoffenheit. Er kultivierte den Mythos eines selbstbestimmten Teils der „Völkerfamilie“, das an der Seite der "schützenden Sowjetunion“ seinen Beitrag als „Friedensstaat“ leistet. Meist waren die Auftritte in einer Art Sympathiekampagne darauf ausgerichtet, kulturell „Weite und Vielfalt“

7 Siehe diese Zuschreibung seit Philip H. Cоомвs, The Forth Dimension of Foreign Policy, New York, Harper and Row, 1964. 
zu suggerieren, das eigene Geltungsbedürfnis zu befriedigen und gleichsam die latenten Inferioritätsempfindungen des kleinen Landes zu kompensieren.

Doch zurück zu den Anfängen: Nach vier Jahren öffentliches Versuchsprogramm hatte das ostdeutsche Telemedium im Januar 1956 als fünftes europäisches Land seinen regulären Betrieb aufgenommen. Zu diesem Zeitpunkt standen auch Dank britischer Exporthilfen mobile Übertragungstechnik sowie Richtfunkstrecken ins Ausland zur Verfügung. Der Ausbau der apparativen Infrastruktur war eine Voraussetzung für den kommenden Programmaustausch.

Die DDR tat sich allerdings schwer, auf internationaler Bühne gleichberechtigt mitzuspielen und den westdeutschen Alleinvertretungsanspruch $\mathrm{zu}$ unterlaufen. Doch gänzlich isoliert werden konnte Ostdeutschland nicht. Denn allein seine geografische Mittellage sozusagen als „Fernseh,Zentralbahnhof' in Europa“(8) zwang beide Seiten zu einem „technokratischen Internationalismus “(9). Ohne politische Grundsätze in Frage zu stellen, waren bei den Frequenzvergaben

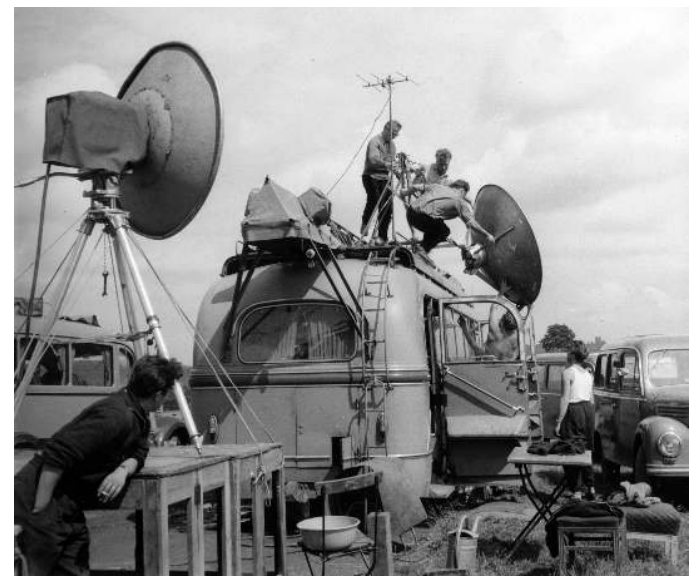

Abb. 4: Mobile Richtfunkanlage für die europaweite Übertragung der Rad-WM in der DDR 1960 (Quelle: Waltraud Denger/DRA Fotobestand) und bei der koordinierten Weiterleitung von bedeutenden Programmen pragmatische Lösungen verlangt. So konnten schon 1956 Bilder von den Olympischen Winterspielen in Cortina d'Ampezzo vom italienischen Fernsehen direkt übernommen und offiziell an einem europäischen TV-Großereignis partizipiert werden.

Seitdem nutzte das DDR-Fernsehen die Kanäle von Intervision und Eurovision für den erforderlichen Import und den angestrebten Export. Darüber hinaus begaben sich die Mitarbeiter für die Programmbeschaffung und zur Vorführung herausgehobener Produktionen direkt zu den Fernsehstationen und Filmlizenzgebern. Oder sie besuchten Fernsehfestivals, Messen und TV-Wettbewerbe. So dienten dem DDR-Fernsehen auch die westeuropäischen Treffen der Medienbranche als Leistungsvergleich, als Orte der gegenseitiger Absprachen und realer An- und Verkäufe. Vertreten war man u.a. auf dem Mercato Internazionale Filme E Documentario (MIFED) in Mailand oder dem Marché International des Programmes de Télévision (MIP-TV) an der Côte d'Azur: „Im Stadtbild Cannes und am Palais waren unter den Fahnen der teilnehmenden Länder auch die Staatsflagge der DDR aufgezogen“, schrieb die Presse 1965 stolz $^{(10)}$. Und die eigenen Sendungen nahmen erfolgreich teil beim Wettbewerb „Goldene Rose von Montreux“, um den „Prix de la Nymphe“ in Monaco sowie am „Prix Jeunesse“ in München.

8 Rudolf VieweG, „DDR wird Fernseh-,Zentralbahnhof“ in Europa“, Lausitzer Rundschau, 10.01.1961.

9 Heiner StAнL, „Suzanne Bernadina Lommers. Europe - On Air. Interwar Projects for Radio Broadcasting“, Rundfunk und Geschichte, Nr. 3-4 (2012), S. 80.

10 „Deutscher Fernsehfunk in Cannes dabei“, Fernsehdienst, Nr. 16 (1965), S. 4. 

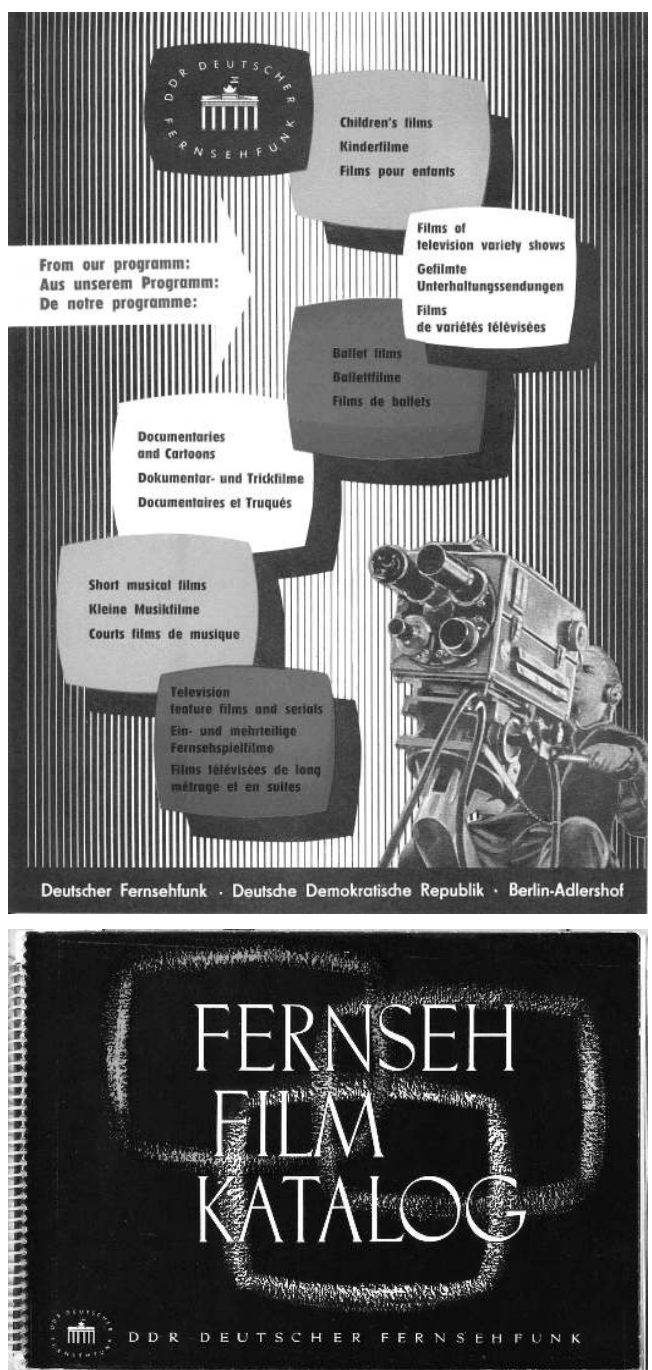

Abb. 5: Internationale Programmwerbung

Deutscher Fernsehfunk 1960er Jahre /

1. Exportkatalog Deutscher Fernsehfunk 1962

(Quelle: DRA SGFS)

Hier wurde die Nähe zu den westlichen TV-Anstalten besonders gesucht. Die Ausweitung des Sendeangebots, die Einführung eines 2. Kanals und des Farbfernsehens hatten den Programmbedarf ständig erhöht. Der war nicht mehr allein mit Eigenproduktionen oder über Zulieferungen der östlichen OIRT-Partner und den zentralen Filmvertrieben im RGWBereich zu decken.

Die Kontakte $\mathrm{zu}$ westlichen TVAnstalten intensivierten sich nach der DDR-Anerkennung und Aufnahme in die UNO 1973. Mitte der 1980er Jahre wurde schließlich mit Sendern in mehr als 70 Ländern zusammengearbeitet und zu über 50 Fernsehorganisationen vertragliche Beziehungen unterhalten ${ }^{(11)}$. Als Beispiel für ein westliches Partnerland sei hier Frankreich angeführt. Der „Grande Nation“ kommt für den Osten nicht nur allgemein eine besondere Rolle in der Zusammenarbeit zu, hatte sie doch im Schulterschluss mit der Sowjetunion das Farbfernsehen und die SatellitenVerbindungen vorangetrieben oder sich als erster und einziger westlicher Staat 1982 am osteuropäischen InterkosmosWeltraumprojekt beteiligt.

Und mit der DDR wurden 1983/84 gegenseitig Kulturzentren in Paris und Ostberlin eingerichtet bzw. 1988 der Besuch von Generalsekretär Honecker bei Staatspräsident Mitterrand hervorgehoben. In diesem Kontext war durch das DDR-Außenministerium sogar angeregt worden, im Fernsehen einen „Sprachkurs französisch“ einzuführen. Aber obwohl über den „politischen Wert [...] selbstverständlich keine Meinungsverschiedenheiten“ bestanden, musste Intendant Adameck den Vorschlag aus Kostengründen ablehnen ${ }^{(12)}$.

11 Kurt Ottersberg, „DDR-Fernsehen für internationale Kooperation“, OIRT Rundfunk und Fernsehen, Nr. 2 (1986), S. 7.

12 Staatliches Komitee für Fernsehen, Adameck, Schreiben an den Leiter der Abteilung Agitation Geggel, Berlin (DDR), 17.11.1987 (BArch DY30-IV2-2.037-42). 
Doch schon früher galt Frankreich als strategischer Bündnispartner, weil sich dort die „kapitalistischen Widersprüche [...] am Schärfsten in der gesellschaftlichen Situation, im Kampf der Klassen und in den Köpfen der Menschen widerspiegeln“ würden. 1957 beispielsweise versuchten Reisekader des Fernsehfunks bei Radiodiffusion-Télévision Française (RTF) und dem PCF-Filmverleih Procinex, „progressive Filme zu erwerben oder mit geeigneten Regisseuren Produktionen durchzuführen“. Eruiert wurden zudem die Möglichkeiten „des Austausches von Fernsehfilmen“, der „Koproduktion für das Fernsehen beim Festival de Cannes“ sowie „eine[s] ständigen Korrespondenten für die [Nachrichtensendung/T.B.] Aktuelle Kamera“ und „eine[s] französischen Korrespondenten, der in Berlin französische Sendungen durchführt “(13). Darüber hinaus stieß ein Vorstoß für den regelmäßigen und kostenneutralen News-Austausch zwischen West und Ost auf Zustimmung, um von den kommerziellen Nachrichtagenturen unabhängiger zu sein. Denn das französische Fernsehen hatte zu diesem Zeitpunkt mit daran gearbeitet,

„ein Informationsbulletin zwischen den wichtigen Fernsehstationen Europas ins Leben zu rufen, das den Sinn hat, kapitalistische Gesellschaften auszuschalten, die mit den Fernsehrechten einen schwunghaften Handel betreiben. Ziel ist es, dass alle beteiligten Fernsehstationen ihre Produktion gegenseitig Meter gegen Meter austauschen und sich in jeder Weise behilflich sind“(14).

Öffentlich in Erscheinung treten konnte das DDR-Fernsehen dann in Paris auf einem Kongress über die Potentiale von Radio und Fernsehen für Erziehung und Bildung unter Schirmherrschaft der EBU/UER im März 1967. Selbstbewusst teilte der „Fernsehdienst“ mit, dass Intendant Adameck vom Generaldirektor des französischen Fernsehens (ORTF) „in einem persönlichen Schreiben“ eingeladen worden war, die DDR „an der Spitze einer offiziellen Delegation“ zu vertreten $^{(15)}$.

Aber auch beim Techniksupport bot sich Frankreich für Ostdeutschland an. Zum einen konnten in den 1950er Jahren dringend benötigte Sendeanlagen des französischen Herstellers Compagnie Générale de Télégraphie Sans Fil (CSF) beschafft werden - allerdings nur auf Umwegen über die Sowjetunion, weil die DDR von den Ausfuhrverboten der westlichen Industrieländer gemäß des geltenden Hochtechnologie-Embargos des Coordinating Committee on Multilateral Export Controls (bzw. for East-West Trade Policy) / COCOM) betroffen war.

Noch enger gestaltete sich die Zusammenarbeit bei der Einführung des Farbfernsehens, das am 20. DDR-Jahrestag 1969 mit einem zweiten Programm gestartet wurde. Mit den östlichen „Bruderländern“ hatte sich die DDR für das französische SECAM-System entschieden, was eine gesamteuropäische Lösung verhinderte. Denn die Entscheidung war gegen eine Kompatibilität mit dem westdeutschen PALSystem gefallen, dem sich die Eurovision-Länder bis auf Frankreich, dem frankophonen Teil Belgiens und Luxemburg angeschlossen hatten. Auf französischer Seite war für Präsident Charles de Gaulle die Durchsetzbarkeit der eigenen Technologie

13 Internationale Redaktion, Thoms, Bericht über meine Reise nach Paris vom 21.2. bis 9.3.1957, Berlin (DDR), 13.03.1957, S. 1f (BArch DR 8/ 415).

14 Ebd., S. 6.

15 „Einladung nach Paris“, Fernsehdienst, Nr. 45 (1966). 
längst „zu einer nationalen Prestigeangelegenheit“ geworden. Das Staatsoberhaupt suchte offensiv die Annäherung an Osteuropa, um seine „Vision ,vom Atlantik bis zum Ural““(16) zu verwirklichen: Ein „Grand Projet“ der politischen Integration mit gesamteuropäischem Blick, mit der er seinen antiamerikanischen Kurs in der Außenpolitik unterstrich.
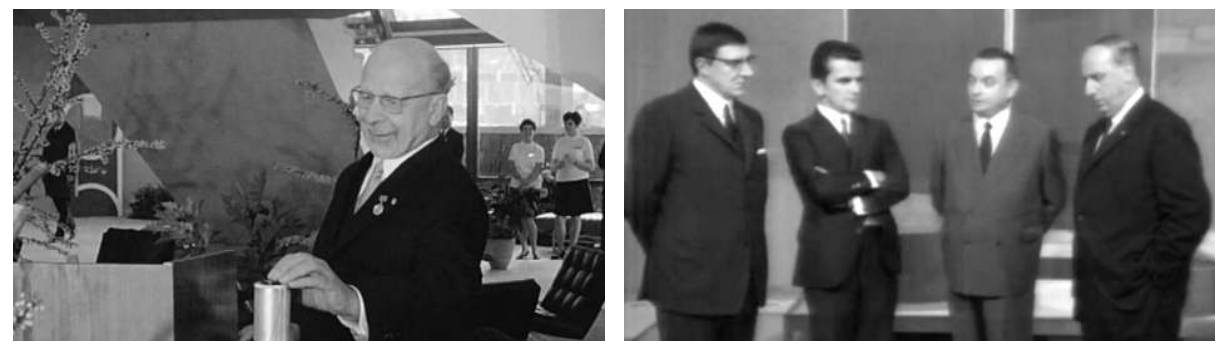

Abb. 6: Screenshots vom Start des Farbfernsehens in der DDR durch Walter Ulbricht 1969 und von der Einführung in Frankreich 1967 im Studio des ORTF

(Quelle: DRA/Mitteldeutscher Rundfunk; INA)

Bei dem Farbfernsehgeschäft erhoffte sich die DDR zusätzlich eine internationale Aufwertung durch ein zwischenstaatliches Abkommen mit Unterzeichnung in Paris. Das wurde durch das französische Außenministerium jedoch „ausdrücklich untersagt“, um der befreundeten Bundesrepublik keine „Möglichkeit zum intervenieren zu geben“(17). Stattdessen kam es zu einer Art Private-Public-Partnership zwischen den zuständigen Organen der DDR und dem Unternehmen CFT. Von staatlicher Seite war Frankreich auf die Wünsche der DDR nur in Bezug auf ein offizielles Begleitschreiben des interministeriellen Delegierten für das Farbfernsehen als Regierungsvertreter eingegangen,

„in dem erklärt wurde, daß der Abschluß [...] mit Befriedigung zur Kenntnis genommen würde und daß sich auf der Grundlage dieses Abkommens fruchtbare Beziehungen entfalten können “(18).

Dem „Abkommen über die Zusammenarbeit auf dem Gebiet des Farbfernsehens“ vom 2. März 1969 folgte ein Lizenzvertrag zwischen CFT und dem VEB RFT Fernsehgerätewerk Staßfurt sowie dem Rundfunk- und Fernsehtechnischen Zentralamt der Deutschen Post. Damit sicherte sich die DDR „die komplexe Übernahme aller Rechte für die Nutzung des Farbfernsehsystems SECAM IIIb“ für eine Gebühr von 1,2 Mio. Francs in vier Jahresraten, die „zum Wareneinkauf durch die französische Seite verwendet werden können“(19).

16 „De Gaulles Fernseh-Allianz“, Die Zeit, Nr. 13 (1965), S. 1

17 Ministerrat, Min. f. Post u. Fernmeldewesen, Bericht über die Verhandlungen mit Vertretern französischen Republik zur Ermittlung der Bedingungen für die Übernahme des Farbfernsehsystems SECAM IIIb seitens in Paris in der Zeit vom 13.-18.1.1969, Berlin (DDR), 20.01.1969, S. 3 und 6 (SAPMO-BArch DY 30/ IV A 2/6.05/ 172).

18 Abt. Verkehr und Verbindungswesen, Mitteilung an Günter Mittag, Berlin (DDR), 10.03.1969 (SAPMO-BArch DY 30/ IV A 2/6.05/ 172).

19 Ministerrat, Min. f. Post u. Fernmeldewesen, Bereich Rundfunk und Fernsehen, Bericht über den Abschluß eines Lizenzvertrages zur Übernahme des Farbfernsehsystems SECAM IIIb durch die DDR 
Neben den Technikspezialisten fuhr ferner eine TV-Delegation an die Seine, um die „in Aussicht gestellten Verbindungen zur französischen Rundfunk- und Fernsehorganisation ORTF herzustellen." Dabei waren

„die Möglichkeiten der Entwicklung des Fernsehprogrammaustausches zwischen beiden Staaten sowie der Austausch ständiger Korrespondenten des Deutschen Fernsehfunks und der ORTF zu untersuchen. [...] Alle Gespräche sind im Geiste des Wunsches nach kultureller Zusammenarbeit zu führen, im Rahmen der Aufgaben, die dem Fernsehen bei der Entwicklung der Beziehungen zwischen der DDR und der Republik Frankreich zufallen. Dabei soll der französischen Seite ein eindrucksvolles Bild von den politischen und kulturellen Leistungen des Fernsehens der DDR im Kampf um Frieden und Völkerfreundschaft vermittelt werden“(20).

Die Initiative schien sich ausgezahlt $\mathrm{zu}$ haben. Denn zu Beginn seiner Farbsendungen griff das DDR-Fernsehen auf französische Importware zurück: Die Zuschauer konnten Konzerte mit namhaften Interpreten wie Joséphine Baker, Charles Aznavour und Enrico Macias im Pariser "Olympia“ miterleben oder den Pantomimen Marcel Marceau und das Stierkampfepos Augenblick der Wahrheit ${ }^{(21)}$ genießen.

Und am 23. November 1976 hatte das DDRFernsehen mit TF1 endlich ein offizielles Abkommen mit längerfristigen Absichtserklärungen zur Förderung des Erfahrungsund Programmaustausches abgeschlossen. Es folgte 1980 der Kanal Antenne 2/A2, der sogleich mit L'Allemande de l'Est eine Art „Themenabend“ in der Reihe A2 pas de chez nous ${ }^{(22)}$ präsentierte.

Insgesamt konnten um die 800 französische TV- und Kino-Streifen akquiriert werden.

Das Interesse reduzierte sich dabei keineswegs nur auf die „Meisterwerke der Filmkunst" wie Außer Atem (À bout de souffle) von Jean-Luc Godard 1975 oder Dokumentarfilme wie Die Welt des Schweigens
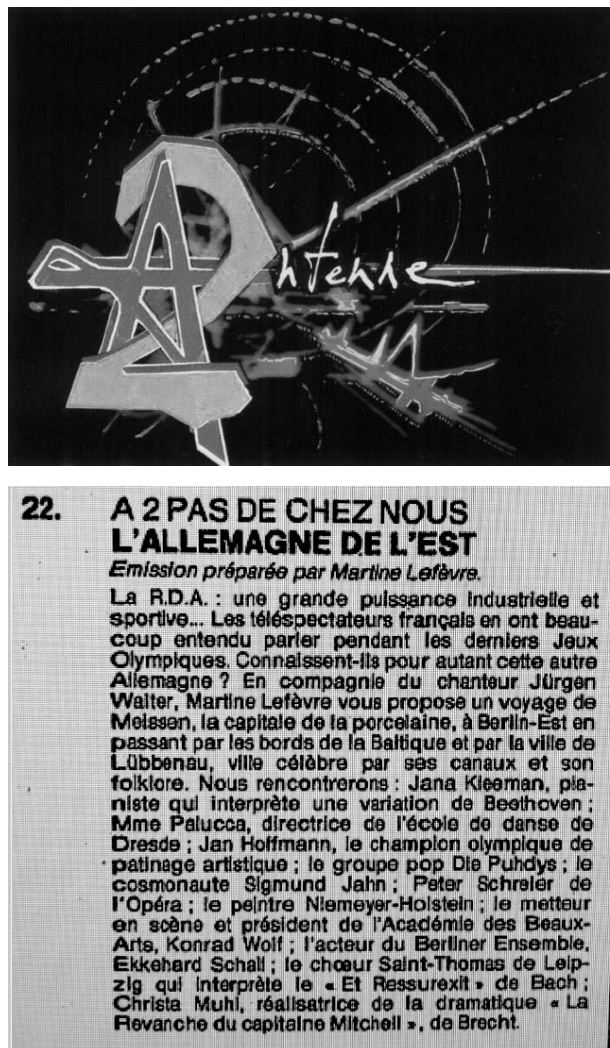

Abb. 7: „Antenne 2“ (1975) / DDR-Programm auf „Antenne 2“ vom 31.08.1980 (Quelle: „Télérama“)

in der Zeit vom 5.-14.3.1969 in Paris, Berlin (DDR), 21.05.1969, S.2 (SAPMO-BArch DY 30/ IV A 2/6.05/ 172).

20 Vorbereitungsgruppe II. Programm - Farbfernsehen, Konzeption für Frankreichreise zur Vorbereitung und Einführung des Farbfernsehens in der DDR, Berlin (DDR), 04.04.1967, S. 2ff (BArch DR 8/ 67).

21 F/I 1965, Regie: Francesco Rosi; siehe „Ausländische Farbfilme“, Fernsehdienst, Sonderausgabe (1969), ohne Seitenangabe.

22 Programmankündigung für den 31.08.1980 in der Rundfunkzeitschrift Télérama. 
(Le Monde du silence) von Jacques Cousteau sogar schon 1957. So begann beispielsweise kurz vor Weihnachten 1986 „unter dem Titel ,Erotisches zur Nacht` zu später Stunde“ laut Programmankündigung eine "sinnenfrohe“ Reihe mit „pikanten Geschichten“ des französischen Fernsehens FR3 nach Motiven von Guy de Maupassant und anderen ${ }^{(23)}$.

Insgesamt standen neben dem Handel mit Filmen vor allem Übertragungen von großen Sportereignissen und Musikkonzerten sowie die aktuelle Berichterstattung im Vordergrund des Programmtransfers. Signifikant ist dabei allerdings das asymmetrische Verhältnis der gegenseitigen Medienübernahmen. Selbst nach den Helsinki-Vereinbarungen der „Konferenz über Sicherheit und Zusammenarbeit in Europa“ (KSZE) über einen erhöhten und ausgewogenen Kulturaustausch 1975 sendete Osteuropa nach UNESCO-Angaben dreimal soviel Programme aus kapitalistischen Ländern wie deren Fernsehanstalten Angebote der anderen Seite ausstrahlten. So beklagte nicht nur die

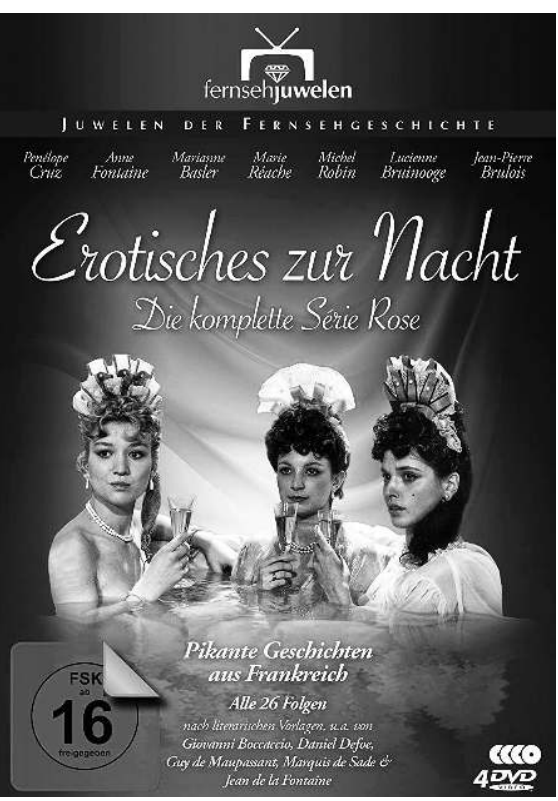

Abb. 8: Erfolgreich vermarktet: die französische TV-Série Rose auch als Kauf-DVD (Quelle: Alive „Fernsehjuwelen“ 1991) DDR einen „Nachholbedarf“ des Westens:

\begin{abstract}
„Wenn da jemand bremst - wir sind es nicht. Es gibt Länder, die ständig nach dem Informationsaustausch rufen, aber nichts dafür tun. Wir senden z.B. bis zu 5o französische Filme jährlich, die Gegenseite einen bis zwei. Ähnlich ist es mit Großbritannien. Wir können das nur als ein Stück politischer Ignoranz gegenüber unbestrittenen Leistungen werten“(24).
\end{abstract}

Die EBU-Mitglieder waren aber nur an unverfänglichen Produktionen interessiert, die hohe Qualitätsstandards aufwiesen wie klassische Musikbeiträge, Literaturverfilmungen oder Kindersendungen. Bei Kommunikaten mit ideologisch aufgeladenen
Growth of the Eurovision-Intervision Program Exchange 1960-1982

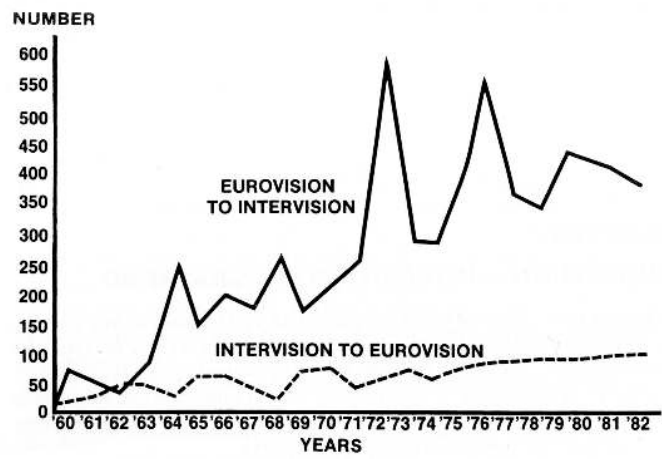

Abb. 9: Die Entwicklung des ungleichen

Programmaustausches zwischen Intervision und Eurovision (Quelle: Statistiken der UER/EBU, OIRT/

Eugster: Television Programming, 1983)

23 „Programminformation ,Erotisches zur Nacht““, Fernsehdienst, Nr. 51 (1986), ohne Seitenangabe; Originalfassung Série Rose (insg. 26 Episoden 1986-91, Sendestart 08.11.1986).

24 Der stellvertretende Programmdirektor Gerd Kaiser, zitiert nach Karl-Claus HaHN, ADN, „Intervisionsländer beraten weiteren Programmaustausch“, Fernseh-Informationen, Nr. 10 (1977), S. 234. 
Sujets und konventionellen Darstellungsmodi hielten sie sich zurück. Das betraf vor allem die redundanten "Generalthemen“ wie der verinnerlichte Antifaschismus, die verklärte Arbeiterbewegung und das Bekenntnis zu den Prinzipien des „Proletarischen Internationalismus“ oder die angebliche Leistungsfähigkeit des planwirtschaftlichen Modells als Ausdruck des zivilisatorischen Fortschritts. Gegenwartsfilme oder Magazine waren nur dann gefragt, wenn sie einen authentischen Einblick in die spezifischen Arbeits- und Lebenswelten des „real existierenden Sozialismus” ermöglichten - sprich: eine mediale Phantasieproduktion und Realitätskonstruktion, die auf zentrale Gesellschaftsfragen oder Herrschaftsdiskurse verweisen und gewisser Maßen eine ,Mental Map' des Ostens konstruieren.

Doch trotz des Ungleichgewichtes im TV-Traffic und trotz der anhaltenden Feindbildproduktion in Form aggressiver „Konterprogramme“ bewirkte der Programmaustausch seit Anfang der sechziger Jahre eine „politico-discursive transformation from security to globalization “(25).

Diese allmähliche Entspannung ermöglichte bei aller kulturpolitischen Negation oder Skepsis gegenüber westlichen Trends einen medienpolitischen Pragmatismus. Das DDR-Fernsehen orientierte sich zunehmend am Weltmarkt. Es versuchte, das eigene Programm anschlussfähig zu machen - mal als simple Imitation, mal als bewusste Adaption und oftmals direkt nach dem Vorbild von ARD und ZDF jenseits der Grenze. Denn die Konsumentenlogik und apolitische Zuschauerbedürfnisse nach

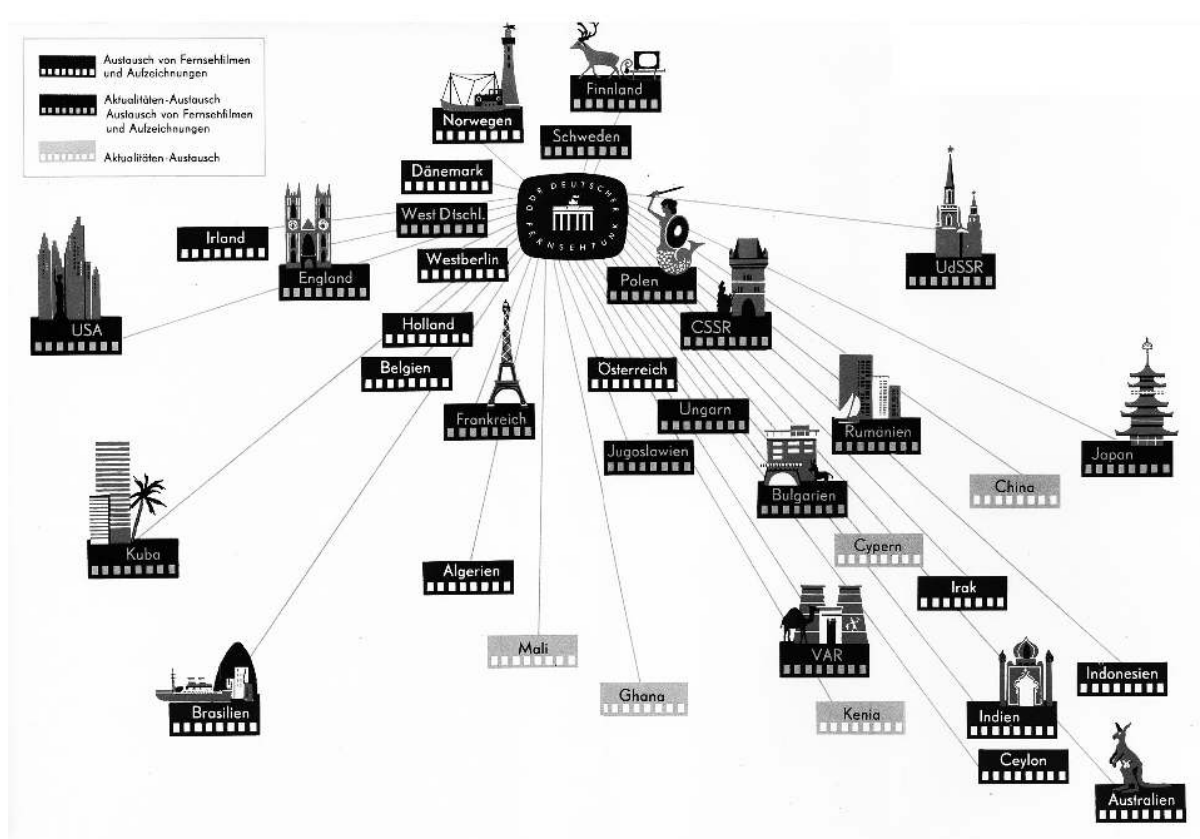

Abb. 10: Internationaler Film- und Nachrichtenaustausch des Deutschen Fernsehfunks Mitte der 1960er Jahre (Quelle: Grafik DFF, Presseabteilung 1964/DRA, SGFS) 
Unterhaltung und Eskapismus mussten immer stärker berücksichtigt werden. Das Ostmedium hatte auf die individualisierte Freizeitgestaltung mit Tendenz zur Privatisierung und latenter Entpolitisierung zu reagieren.

Darüber hinaus richtete es sich an internationalen Standards aus, um den hedonistischen Versprechen der „Agenturen der liberalen Konsumgesellschaft“(26) etwas annähernd Gleichwertiges entgegensetzen zu können. Die Anpassung an westliche Medienstrategien zeigte sich besonders an den wachsenden Ankäufen von Kino- und TV-Filmen ausländischer Provenienz; galt es doch, mit bewährten, aber nicht selbst bedienten Genres dem Zeitgeist und den Moden zu folgen. Über mehr Action und Komik, beliebte Darsteller und spektakuläre Schauplätze sollte eine engere Zuschauerbindung erreicht werden. Durch diesen gezielten Input bot das Abendprogramm dann in den achtziger Jahren ein erstaunliches Bild: nur $40 \%$ an Eigensendungen standen mit $60 \%$ wesentlich mehr Fremdproduktionen ${ }^{(27)}$ gegenüber.

Mit den eingeleiteten Maßnahmen wurde auf Dauer aber nicht nur eine Diversifizierung des Angebots erreicht. Sie haben wie im Westen - wenn auch zeitverzögert - in der Tendenz zu „Uniformität und Schematismus“(28) beigetragen und zu einer allmählichen Konvergenz mit anderen europäischen Fernsehnationen geführt. Als Analogien zu erkennen sind:

- das klar strukturierte Sendeschema mit bevorzugter Primetime, definierten Programmplätzen, angeglichenen Präsentationsmustern und starker Formatisierung;

- die angestrebte Zuschauerbindung („Audience Flow“) durch die Kopplung publikumswirksamer Angebote;

- die Bedienung des Mainstreams statt bildungsbürgerlicher Ambitionen;

- die zunehmende Bedeutung von Unterhaltungsoffensiven und die Aufwertung von Bildschirmstars;

- industrialisierte Produktionsstandards, die Intensivierung der Serienproduktion, die Marktorientierung (Export) und die verstärkte Filmauswertung (Import).

Hier ist also eine sichtbare Implementierung des ,Fremden' zu beobachten, mit der sich narrative Muster und formales Repertoire der kapitalistischen Unterhaltungsindustrie in das kulturelle Feld der DDR eingeschrieben haben. Es ist auffällig, dass die zuständigen Kontrollinstanzen diese für sie sicherlich erkennbare Aufweichung des ursprünglichen kulturpolitischen Anspruchs mit der Zeit hinnahmen und ein so auffällig disparates Spektrum durchgehen ließen. Sah man sich doch mit Fremdanleihen konfrontiert, die zwar einen hohen Attraktionswert besaßen. Aber sie konnten inhaltlich und formal kaum mit den Vorstellungen von einer „sozialistischen Massenkultur“ kompatibel sein. Gebilligt wurden sie trotzdem, um die erkannten Programmdefizite und fehlende Produktionskapazitäten zu kompensieren. Darüber hinaus stand mit Beginn der Honecker-Ära auch ein gesellschaftlicher Innovationsanspruch nach der

26 Christoph Classen, „DDR-Medien im Spannungsfeld von Gesellschaft und Politik“, in: Stefan ZAHLMANN (Hg.), Wie im Westen, nur anders. Medien in der DDR, Berlin, Panama Verlag, 2010, S. 407.

27 Nach internen Berechnungen Erich Selbmann, DFF Adlershof: Wege übers Fernsehland. Zur Geschichte des DDR-Fernsehens, Berlin, Edition Ost, 1998, S. 364.

28 Siehe dazu Gerhard Eckert, Das Fernsehen in den Ländern Westeuropas: Entwicklung und gegenwärtiger Stand, Gütersloh, Bertelsmann, 1965. 
bekannten Maxime „Einheit von Wirtschafts- und Sozialpolitik“im Vordergrund auch wenn der wiederum spätestens in den 1980 Jahren in eine ideologische Orientierungslosigkeit umschlug - oder, wie es Manfred Jäger treffend formuliert hat: in eine "gewollte und praktizierte Haltung des Abwartens und Aussitzens“(29).

Angesichts der massiven Rückgriffe auf die Bilder und Töne des Klassenfeindes konnte „das normative Projekt des Westens “(30) kaum noch glaubwürdig in Frage gestellt werden. Es fanden sich keine überzeugenden Mittel gegen jene Phalanx pluralistischer Demokratien, die mit Meinungsfreiheit und Menschenrecht, mit marktwirtschaftlicher Potenz und individuellem Lebensstil warben und überzeugten.

Dabei werden einmal mehr die Widersprüche deutlich, die sich aus der makropolitischen Doppelstrategie des östlichen Bündnisses ergeben hatten: der Abgrenzung von Westeuropa als eine „imperialistische Machtbasis“ einerseits und der Annäherung in Form „friedlicher Koexistenz“ andererseits. In dem bipolaren Weltbild verschwamm nun zusehends die Trennlinie zwischen unerwünschten oder tolerablen Programmen. Die einen galten nach Lesart der SED als negativer Ausdruck des „Kulturverfalls“ oder der „ideologischen Diversion“, der „,antikommunistischen Hetze“ und der „Einmischung in innere Angelegenheiten“. Die anderen Programme hingegen konnten mit der eigenen Vorstellung von „humanistischem Gedankengut“ und „progressivem Kulturerbe“ oder „Völkerverständigung“ und „Friedensicherung“ in Einklang gebracht werden.

Wie sind nun der Umfang und die präferierten Genres des televisionären Transfers letztlich einzuordnen? Ich schließe mich der These an, dass sich diese transkulturelle Kommunikation weniger als dialogischer Austauschprozess, sondern vielmehr als eine Akkulturation bzw. „Überschreitung“ im Verständnis von Michel Foucault darstellt. Kann nicht im Fall des DDR-Fernsehens von einer schleichenden ,Verwestlichung' gesprochen werden, die einer, Sowjetisierung' erfolgreich entgegenwirkte ${ }^{(31)}$ ? In wieweit repräsentierte die eigene Medienkultur dann überhaupt noch den auferlegten sozialistischen „Weltentwurf“?

Grundsätzlich lässt sich zumindest festhalten, dass sich die Strategien der medialen Konfrontation und Agitation durch den Programmtransfer hin zu einer sukzessiven Dialogbereitschaft und Annäherung verlagert haben. Doch die mediale Transformation und Extension durch die potenzierte Zunahme der Übernahmen scheinen weniger entspannungspolitischen Zielen oder einer europäischen Annäherung geschuldet zu sein. Sie sind wohl eher auf den hohen Wettbewerbsdruck zurückzuführen, der durch das empfangbare Westfernsehen von Beginn an spürbar war.

Zudem lösten die dauerhaften Wechselbeziehungen beim DDR-Fernsehen einen frühen Synchronisierungsprozess mit Tendenz zu einer,Cross-Culture` aus - auch

29 Manfred Jäger, Kultur und Politik in der DDR. 1945-1990, Köln, Edition Deutschland Archiv, 1995 , S. 210 .

30 Heinrich August Winkler, Geschichte des Westens. Von den Anfängen in der Antike bis zum 20. Jahrhundert, München, C.H. Beck, 2009, S.24.

31 Anselm Doering-Manteuffel, „Amerikanisierung und Westernisierung“, Docupedia-Zeitgeschichte, 18.01.2011, docupedia.de/zg/Amerikanisierung_und_Westernisierung?oldid=125786 (letzter Abruf: 22. März 2019). 
wenn die partielle Öffnung keinen Beitrag zu einer echten Partizipation oder einer gesellschaftlichen Streitkultur leisten konnte. Nach Thomas Lindenberger konnte aber die Verständigung und Vernetzung der Sender zumindest die tradierte „Dichotomi-

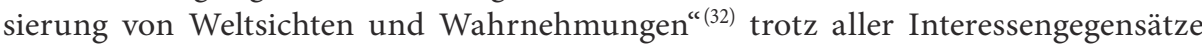
aufweichen. Ist dadurch nicht bereits vor 1990 ansatzweise eine gesamteuropäische Medienöffentlichkeit als „virtuelle Gemeinschaft“(33) in einem immer durchlässigeren Kommunikationsraum entstanden? Das hat - wie es Christian Henrich-Franke abschließend formuliert - zwar weniger ein europäisches Bewusstsein oder eine europäische Identität geschaffen, aber zumindest ein Bewusstsein für Europa erzeugt ${ }^{(34)}$.

\section{Zusammenfassung}

Die ostdeutsche Fernsehlandschaft erscheint im Kontext des Cold War CultureDiskurses untersuchungsrelevant. Die DDR hatte nicht nur aufgrund des permanenten Konkurrenzverhältnisses zur Bundesrepublik eine Sonderrolle. Sie war auch ein ,Musterland' mit hoher Anpassungsbereitschaft in der sozialistischen Staatengemeinschaft. Im kontinentalen Medienverkehr an der Nahtstelle und Bruchzone der Blöcke Ost und West fungierte die DDR zudem als wichtiges Transitland. Darüber hinaus sind sowohl vielseitige internationale Beziehungen zu beobachten als auch ein grenz- und systemübergreifender Programmtransfer. Er wurde nach dem Ausbau der RichtfunkSendenetze schon seit 1956 rege genutzt und von unterschiedlichen TV-Kulturen aus beiden Lagern gespeist.

Seit den siebziger Jahren arbeitete das DDR-TV mit Sendern in mehr als 70 Ländern zusammen und unterhielt zu über 50 Fernsehorganisationen vertragliche Beziehungen. Die externen Verbindungen und die sichtbare Medienpräsenz im Ausland erwiesen sich vor allem in dem geführten Kampf um völkerrechtliche Anerkennung als sehr produktiv. Auf diese Weise konnte reale Außenpolitik betrieben sowie das eigene Geltungsbedürfnis befriedigt werden.

\section{Abstract}

East German television seems an appropriate subject of study in the context of the discurs of cold war culture. The GDR was not only unique for its permanent state of competition with the Federal Republic. It was also an ideal model with strong conformist tendencies in the socialist community of states. Located on the boundary and fault line of the Eastern and Western blocs, the GDR also served as an important transit country. It likewise had wide-ranging international relations and a cross-border, cross-system

32 Thomas Lindenberger, „Geteilte Welt, geteilter Himmel. Der Kalte Krieg und die Massenmedien in gesellschaftsgeschichtlicher Perspektive“, in: Klaus Arnold, Christoph Classen (Hg.), Zwischen Pop und Propaganda. Radio in der DDR, Berlin, 2004, p. 33.

33 Jens Ruchatz, „Spiel ohne Grenzen oder grenzenlose Spielerei? Eurovision - Intervision - Mondovision“, in: Irmela Schneider, Torsten Hahn, Christina Bartz (Hg.), Medienkultur der 60er Jahre. Global - lokal, Wiesbaden, 2003 (=Diskursgeschichte der Medien nach 1945, Bd. 2), p. 124.

34 Christian Henrich-Franke, „Eurovision. Europäischer Fernsehprogrammaustausch in seinen Anfangsjahren“, in: Themenportal Europäische Geschichte, 2016, www.europa.clio-online.de/essay/id/ artikel-3753. 
transfer of television programs. This exchange was actively used after the expansion of radio-relay networks in 1956, and was fed from a variety of television cultures from both sides.

From the 1970s on, GDR-Television was cooperating with more than 70 countries and had contractual relations with over 50 television organizations. These external relations and its visible media presence abroad proved to be highly effective in its struggle for international recognition outside of the Soviet sphere of influence. They were a concrete way of exercising foreign policy while at the same time satisfying its desire to be taken seriously. 